\title{
New Class of Almost Unbiased Modified Ratio Cum Product Estimators with Knownparameters of Auxiliary Variables
}

\author{
Jambulingam Subramani and Master Ajith S \\ Department of Statistics \\ Ramanujan School of Mathematical sciences \\ Pondicherry University \\ Puducherry, 605014, India \\ Email id: drjsubramani@yahoo.co.in and ajith.master9@gmail.com
}

\begin{abstract}
This manuscript deals with new class of almost unbiased ratio cum product estimators for the estimation of population mean of the study variable by using the known values auxiliary variable. The bias and mean squared error of proposed estimators are obtained. An empirical study is carried out to assess the efficiency of proposed estimators over the existing estimators with the help of some known natural populations and it shows that the proposed estimators are almost unbiased and it perform better than the existing estimators.
\end{abstract}

Keywords: Auxiliary variable, Bias, Mean squared error ,Natural populations, Ratio and Product estimators, Simple random sampling .

\section{Introduction}

The efficiency of the estimators of the population parameters can be increased by using the prior information of the study characteristics. In literature several estimators exist with auxiliary variables involved. The commonly used thepopulation parameters of the auxiliary variables are mean, median, coefficient of variation, coefficient of skewness,coefficient of kurtosis etc. Ratio method of estimation is extensively used because of its computational simplicity and applicability. The correlation between study and auxiliary variables are negative the product method of estimation is used. Several researchers have directed their efforts towards to get efficient estimators of population mean. These estimators are biased but the percentage relative efficiency is better than that of simple random sampling, ratio and product estimators. For this reason, we consider the problem of estimation of population mean of study variable using known values of the auxiliary variable. So we have suggested new class modified ratio cum product estimators for estimating the population mean of the study variable. To know more abouthistorical developments of the estimation of population mean are referred to Cochran[1,2], Subramani and Master Ajith [4,5], Murthy[7,8],Subramani[14], Subramani and kumarapandiyan[15,16,17], Upadhyaya and Singh[18], Yan and Tian[19] and the references cited there in.

Consider a finite population $\mathrm{U}$ of size $\mathrm{N}$ consisting of $U_{1}, U_{2}, U_{3} \ldots U_{N}$ units. Each $\mathrm{U}_{\mathrm{i}}=\left(\mathrm{X}_{\mathrm{i}}, \mathrm{Y}_{\mathrm{i}}\right),(\mathrm{i}$ $=1,2,3 \ldots . \mathrm{N}$ ) has a pair of values. Here $\mathrm{Y}$ be the study variable and $\mathrm{X}$ be the auxiliary variable which is correlated with Y. If $y=\left\{y_{1}, y_{2}, y_{3} \ldots y_{n}\right\}$, and $x=\left\{x_{1}, x_{2}, x_{3} \ldots x_{n}\right\}$ be $n$ sample values. Let $\bar{y}$ and $\bar{x}$ be the sample means of the study and auxiliary variables, $S_{y}{ }^{2}=\frac{1}{N-1} \sum_{i=1}^{N}\left(Y_{i}-\bar{Y}\right)^{2}, S_{x}{ }^{2}=$ $\frac{1}{N-1} \sum_{i=1}^{N}\left(X_{i}-\bar{X}\right)^{2}$ and $S_{x y}=\frac{1}{N-1} \sum_{i=1}^{N}\left(Y_{i}-\bar{Y}\right)\left(X_{i}-\bar{X}\right)$ be the population variance and covariance of the study variable and auxiliary variable. Similarly the coefficient of variations and 
coefficient of co-varianceof these variables are defined as $C_{y}=\frac{S_{y}}{\bar{Y}}, C_{x}=\frac{S_{x}}{\bar{X}}$ and $C_{x y}=\frac{S_{x y}}{\bar{X} \bar{Y}}=$ $\rho C_{x} C_{y}$ where $\rho$ is the correlation coefficient

The simple random sample mean without replacement is used only when there is no additional information of the study variable is available,. In simple random sampling without replacement the estimator of population mean $\bar{y}_{s r s}$ is an unbiased estimator for the population mean. The auxiliary variable and study variable are positively correlated,Cochran [1]introduced ratio estimator for the estimation of population mean and it is given by

$$
\hat{\bar{Y}}_{R}=\frac{\bar{y}}{\bar{x}} \overline{\mathrm{X}}=\hat{R} \bar{X}
$$

the bias and mean squared error of ratio estimator up to first order approximations are

$$
\begin{gathered}
\mathrm{B}\left(\hat{\bar{Y}}_{R}\right)=\delta \bar{Y}\left[C_{x}{ }^{2}-\rho C_{x} C_{y}\right] \\
\operatorname{MSE}\left(\hat{\bar{Y}}_{R}\right)=\delta \bar{Y}^{2}\left[C_{y}{ }^{2}+C_{x}{ }^{2}-2 \rho C_{x} C_{y}\right]
\end{gathered}
$$

Where $\delta=\left(\frac{1-\mathrm{f}}{\mathrm{n}}\right), f=\frac{n}{N}$

\begin{tabular}{|c|c|c|c|}
\hline Existing Estimators & Constants & Bias & Variance/Mean squared Error \\
\hline $\begin{array}{l}\hat{Y}_{M R 1}=\bar{y}\left(\frac{C_{x} \bar{X}+\beta_{1}}{C_{x} \bar{x}+\beta_{1}}\right) \\
\text { Yan and Tian [19] }\end{array}$ & $\theta_{1}=\frac{C_{x} \bar{X}}{C_{x} \bar{X}+\beta_{1}}$ & $\mathrm{~B}\left(\hat{\bar{Y}}_{M R 1}\right)=\delta \bar{Y}\left[\theta_{1}^{2} C_{x}^{2}-\theta_{1} \rho \mathrm{C}_{\mathrm{x}} \mathrm{C}_{\mathrm{y}}\right]$ & $\operatorname{MSE}\left(\hat{\bar{Y}}_{M R 1}\right)=\delta \bar{Y}^{2}\left[C_{y}^{2}+\theta_{1}^{2} C_{x}^{2}-2 \theta_{1} \rho C_{x} C_{y}\right]$ \\
\hline$\hat{Y}_{M R 2}=\bar{y}\left(\frac{\beta_{1} \bar{X}+C_{x}}{\beta_{1} \bar{x}+C_{x}}\right)$ & $\theta_{2}=\frac{\beta_{1} \bar{X}}{\beta_{1} \bar{X}+C_{x}}$ & $\mathrm{~B}\left(\hat{\bar{Y}}_{M R 2}\right)=\delta \bar{Y}\left[\theta_{2}^{2} C_{x}^{2}-\theta_{2} \rho C_{x} C_{y}\right]$ & $\operatorname{MSE}\left(\hat{\bar{Y}}_{M R 2}\right)=\delta \bar{Y}^{2}\left[C_{y}{ }^{2}+\theta_{2}{ }^{2} C_{x}{ }^{2}-2 \theta_{2} \rho C_{x} C_{y}\right]$ \\
\hline$\hat{\bar{Y}}_{M R 3}=\bar{y}\left(\frac{C_{x} \bar{X}+\rho}{C_{x} \bar{x}+\rho}\right)$ & $\theta_{3}=\frac{C_{x} \bar{X}}{C_{x} \bar{X}+\rho}$ & $\mathrm{B}\left(\hat{\bar{Y}}_{M R 3}\right)=\delta \bar{Y}\left[\theta_{3}^{2} C_{x}^{2}-\theta_{3} \rho C_{x} C_{y}\right]$ & $\operatorname{MSE}\left(\hat{\bar{Y}}_{M R 3}\right)=\delta \bar{Y}^{2}\left[C_{y}{ }^{2}+\theta_{3}{ }^{2} C_{x}{ }^{2}-2 \theta_{3} \rho C_{x} C_{y}\right]$ \\
\hline$\hat{\bar{Y}}_{M R 4}=\bar{y}\left(\frac{\rho \bar{X}+C_{x}}{\rho \bar{x}+C_{x}}\right)$ & $\theta_{4}=\frac{\rho \bar{X}}{\rho \bar{X}+C_{x}}$ & $\mathrm{~B}\left(\hat{\bar{Y}}_{M R 4}\right)=\delta \bar{Y}\left[\theta_{4}^{2} C_{x}^{2}-\theta_{4} \rho \mathrm{C}_{\mathrm{x}} \mathrm{C}_{\mathrm{y}}\right]$ & $\operatorname{MSE}\left(\hat{\bar{Y}}_{M R 4}\right)=\delta \bar{Y}^{2}\left[C_{y}{ }^{2}+\theta_{4}{ }^{2} C_{x}{ }^{2}-2 \theta_{4} \rho \mathrm{C}_{\mathrm{x}} \mathrm{C}_{\mathrm{y}}\right]$ \\
\hline $\begin{array}{c}\hat{Y}_{M R 5}=\bar{y}\left(\frac{C_{x} \bar{X}+\beta_{2}}{C_{x} \bar{x}+\beta_{2}}\right) \\
\text { Upadhyaya and Singh [18] }\end{array}$ & $\theta_{5}=\frac{C_{x} \bar{X}}{C_{x} \bar{X}+\beta_{2}}$ & $\mathrm{~B}\left(\hat{\bar{Y}}_{M R 5}\right)=\delta \bar{Y}\left[\theta_{5}^{2} C_{x}^{2}-\theta_{5} \rho \mathrm{C}_{\mathrm{x}} \mathrm{C}_{\mathrm{y}}\right]$ & $\operatorname{MSE}\left(\hat{\bar{Y}}_{M R 5}\right)=\delta \bar{Y}^{2}\left[C_{y}{ }^{2}+\theta_{5}^{2} C_{x}^{2}-2 \theta_{5} \rho C_{x} C_{y}\right]$ \\
\hline $\begin{array}{c}\hat{\bar{Y}}_{M R 6}=\bar{y}\left(\frac{\beta_{2} \bar{X}+C_{x}}{\beta_{2} \bar{x}+C_{x}}\right) \\
\text { Upadhyaya and Singh [18] }\end{array}$ & $\theta_{6}=\frac{\beta_{2} \bar{X}}{\beta_{2} \bar{X}+C_{x}}$ & $\mathrm{~B}\left(\hat{\bar{Y}}_{M R 6}\right)=\delta \bar{Y}\left[\theta_{6}^{2} C_{x}^{2}-\theta_{6} \rho \mathrm{C}_{\mathrm{x}} \mathrm{C}_{\mathrm{y}}\right]$ & $\operatorname{MSE}\left(\hat{\bar{Y}}_{M R 6}\right)=\delta \bar{Y}^{2}\left[C_{y}^{2}+\theta_{6}^{2} C_{x}^{2}-2 \theta_{6} \rho C_{x} C_{y}\right]$ \\
\hline
\end{tabular}

Some existing modified ratio estimators with their biases and mean squared errors are in table 1

Table 1: Bias and MSE of Existing modified ratio estimators

The auxiliary variable and study variable are negatively correlated, product estimator (Murthy[7]) is used,the bias and mean squared error of product estimator up to first order approximations are

$$
\begin{gathered}
\mathrm{B}\left(\hat{\bar{Y}}_{R}\right)=\delta \bar{Y}\left[\rho C_{x} C_{y}\right] \\
\operatorname{MSE}\left(\hat{\bar{Y}}_{R}\right)=\delta \bar{Y}^{2}\left[C_{y}{ }^{2}+C_{x}{ }^{2}+2 \rho C_{x} C_{y}\right]
\end{gathered}
$$


New Class of Almost Unbiased Modified Ratio Cum Product Estimators with Knownparameters of Auxiliary Variables

\begin{tabular}{|l|l|c|l|}
\hline $\begin{array}{l}\text { Existing } \\
\text { Estimators }\end{array}$ & Constants & Bias & Variance/Mean squared Error \\
\hline$\hat{Y}_{M P 1}=\bar{y}\left(\frac{C_{x} \bar{x}+\beta_{1}}{C_{x} \bar{X}+\beta_{1}}\right)$ & $\theta_{1}=\frac{C_{x} \bar{X}}{C_{x} \bar{X}+\beta_{1}}$ & $\mathrm{~B}\left(\hat{\bar{Y}}_{M P 1}\right)=\delta \bar{Y}\left[\theta_{1} \rho \mathrm{C}_{\mathrm{x}} \mathrm{C}_{\mathrm{y}}\right]$ & $\operatorname{MSE}\left(\hat{\bar{Y}}_{M P 1}\right)=\delta \bar{Y}^{2}\left[C_{y}{ }^{2}+\theta_{1}{ }^{2} C_{x}{ }^{2}+2 \theta_{1} \rho \mathrm{C}_{\mathrm{x}} \mathrm{C}_{\mathrm{y}}\right]$ \\
\hline$\hat{\bar{Y}}_{M P 2}=\bar{y}\left(\frac{\beta_{1} \bar{x}+C_{x}}{\beta_{1} \bar{X}+C_{x}}\right)$ & $\theta_{2}=\frac{\beta_{1} \bar{X}}{\beta_{1} \bar{X}+C_{x}}$ & $\mathrm{~B}\left(\hat{\bar{Y}}_{M P 2}\right)=\delta \bar{Y}\left[\theta_{2} \rho \mathrm{C}_{\mathrm{x}} \mathrm{C}_{\mathrm{y}}\right]$ & $\operatorname{MSE}\left(\hat{\bar{Y}}_{M P 2}\right)=\delta \bar{Y}^{2}\left[C_{y}{ }^{2}+\theta_{2}{ }^{2} C_{x}{ }^{2}+2 \theta_{2} \rho \mathrm{C}_{\mathrm{x}} \mathrm{C}_{\mathrm{y}}\right]$ \\
\hline$\hat{\bar{Y}}_{M P 3}=\bar{y}\left(\frac{C_{x} \bar{x}+\rho}{C_{x} \bar{X}+\rho}\right)$ & $\theta_{3}=\frac{C_{x} \bar{X}}{C_{x} \bar{X}+\rho}$ & $\mathrm{B}\left(\hat{Y}_{M P 3}\right)=\delta \bar{Y}\left[\theta_{3} \rho \mathrm{C}_{\mathrm{x}} \mathrm{C}_{\mathrm{y}}\right]$ & $\operatorname{MSE}\left(\hat{\bar{Y}}_{M P 3}\right)=\delta \bar{Y}^{2}\left[C_{y}{ }^{2}+\theta_{3}{ }^{2} C_{x}{ }^{2}+2 \theta_{3} \rho \mathrm{C}_{\mathrm{x}} \mathrm{C}_{\mathrm{y}}\right]$ \\
\hline$\hat{\bar{Y}}_{M P 4}=\bar{y}\left(\frac{\rho \bar{x}+C_{x}}{\rho \bar{X}+C_{x}}\right)$ & $\theta_{4}=\frac{\rho \bar{X}}{\rho \bar{X}+C_{x}}$ & $\mathrm{~B}\left(\hat{\bar{Y}}_{M P 4}\right)=\delta \bar{Y}\left[\theta_{4} \rho \mathrm{C}_{\mathrm{x}} \mathrm{C}_{\mathrm{y}}\right]$ & $\operatorname{MSE}\left(\hat{\bar{Y}}_{M P 4}\right)=\delta \bar{Y}^{2}\left[C_{y}{ }^{2}+\theta_{4}{ }^{2} C_{x}{ }^{2}+2 \theta_{4} \rho \mathrm{C}_{\mathrm{x}} \mathrm{C}_{\mathrm{y}}\right]$ \\
\hline$\hat{\bar{Y}}_{M P 5}=\bar{y}\left(\frac{C_{x} \bar{x}+\beta_{2}}{C_{x} \bar{X}+\beta_{2}}\right)$ & $\theta_{5}=\frac{C_{x} \bar{X}}{C_{x} \bar{X}+\beta_{2}}$ & $\mathrm{~B}\left(\hat{\bar{Y}}_{M P 5}\right)=\delta \bar{Y}\left[\theta_{5} \rho \mathrm{C}_{\mathrm{x}} \mathrm{C}_{\mathrm{y}}\right]$ & $\operatorname{MSE}\left(\hat{\bar{Y}}_{M P 3}\right)=\delta \bar{Y}^{2}\left[C_{y}{ }^{2}+\theta_{5}{ }^{2} C_{x}{ }^{2}+2 \theta_{5} \rho \mathrm{C}_{\mathrm{x}} \mathrm{C}_{\mathrm{y}}\right]$ \\
\hline$\hat{Y}_{M P 6}=\bar{y}\left(\frac{\beta_{2} \bar{x}+C_{x}}{\beta_{2} \bar{X}+C_{x}}\right)$ & $\theta_{6}=\frac{\beta_{2} \bar{X}}{\beta_{2} \bar{X}+C_{x}}$ & $\mathrm{~B}\left(\hat{\bar{Y}}_{M P 6}\right)=\delta \bar{Y}\left[\theta_{6} \rho \mathrm{C}_{\mathrm{x}} \mathrm{C}_{\mathrm{y}}\right]$ & $\operatorname{MSE}\left(\hat{\bar{Y}}_{M P 2}\right)=\delta \bar{Y}^{2}\left[C_{y}{ }^{2}+\theta_{6}{ }^{2} C_{x}{ }^{2}+2 \theta_{6} \rho \mathrm{C}_{\mathrm{x}} \mathrm{C}_{\mathrm{y}}\right]$ \\
\hline
\end{tabular}

Some existing modified product estimators with their biases and mean squared errors are given in table 2

Table 2: Bias and MSE of Existing modified product estimators

\section{Suggested Class of Estimators}

In this section, new class of modified ratio cum product estimators for the population mean by using the known parameters of auxiliary variableis proposed and also derived the bias and the mean squared errors of the proposed estimators.

The proposed estimators are given by

$$
\begin{gathered}
\widehat{\bar{Y}}_{P 1}=\alpha_{1} \lambda_{1} \bar{y}\left(\frac{C_{x} \bar{X}+\beta_{1}}{C_{x} \bar{x}+\beta_{1}}\right)+\left(1-\alpha_{1}\right) \gamma_{1} \bar{y}\left(\frac{C_{x} \bar{x}+\beta_{1}}{C_{x} \bar{X}+\beta_{1}}\right) \\
\widehat{\bar{Y}}_{P 2}=\alpha_{2} \lambda_{2} \bar{y}\left(\frac{\beta_{1} \bar{X}+C_{x}}{\beta_{1} \bar{x}+C_{x}}\right)+\left(1-\alpha_{2}\right) \gamma_{2} \bar{y}\left(\frac{\beta_{1} \bar{x}+C_{x}}{\beta_{1} \bar{X}+C_{x}}\right) \\
\widehat{\bar{Y}}_{P 3}=\alpha_{3} \lambda_{3} \bar{y}\left(\frac{C_{x} \bar{X}+\rho}{C_{x} \bar{x}+\rho}\right)+\left(1-\alpha_{3}\right) \gamma_{3} \bar{y}\left(\frac{C_{x} \bar{x}+Q_{r}}{C_{x} \bar{X}+Q_{r}}\right) \\
\widehat{\bar{Y}}_{P 4}=\alpha_{4} \lambda_{4} \bar{y}\left(\frac{\rho \bar{X}+C_{x}}{\rho \bar{x}+C_{x}}\right)+\left(1-\alpha_{4}\right) \gamma_{4} \bar{y}\left(\frac{\rho \bar{x}+C_{x}}{\rho \bar{X}+C_{x}}\right) \\
\widehat{\bar{Y}}_{P 5}=\alpha_{5} \lambda_{5} \bar{y}\left(\frac{C_{x} \bar{X}+\beta_{2}}{C_{x} \bar{x}+\beta_{2}}\right)+\left(1-\alpha_{5}\right) \gamma_{5} \bar{y}\left(\frac{C_{x} \bar{x}+\beta_{2}}{C_{x} \bar{X}+\beta_{2}}\right) \\
\widehat{\bar{Y}}_{P 6}=\alpha_{6} \lambda_{6} \bar{y}\left(\frac{\beta_{2} \bar{X}+C_{x}}{\beta_{2} \bar{x}+C_{x}}\right)+\left(1-\alpha_{6}\right) \gamma_{6} \bar{y}\left(\frac{\beta_{2} \bar{x}+C_{x}}{\beta_{2} \bar{X}+C_{x}}\right)
\end{gathered}
$$

Where $\lambda_{i}=\frac{s_{y}}{S_{y}+a_{i} C_{y}} \gamma_{i}=\frac{S_{y}}{S_{y}+b_{i} C_{y}}, \mathrm{i}=1,2,3,4,5,6$. Here $a_{i}$ 'sand $b_{i}$ 's are constants. $S_{y}$ and $C_{y}$ are the population variance and coefficient of variation of study variable respectively. It is reasonable to assume that the values of $S_{y}$ and $C_{y}$ are known from the previous studies.

\subsection{The Bias and Mean Squared error of the Proposed Estimator}


To obtain the bias and mean squared error of the proposed estimator, Consider

$$
\begin{gathered}
e_{0}=\frac{\bar{y}-\bar{Y}}{\bar{Y}}, e_{1}=\frac{\bar{x}-\bar{X}}{\bar{X}}, \\
\theta_{1}=\frac{C_{x} \bar{X}}{C_{x} \bar{X}+\beta_{1}}, \theta_{2}=\frac{\beta_{1} \bar{X}}{\beta_{1} \bar{X}+C_{x}} \theta_{3}=\frac{C_{x} \bar{X}}{C_{x} \bar{X}+\rho}, \theta_{4}=\frac{\rho \bar{X}}{\rho \bar{X}+C_{x}}, \theta_{5}=\frac{C_{x} \bar{X}}{C_{x} \bar{X}+\beta_{2}}, \theta_{6}=\frac{\beta_{2} \bar{X}}{\beta_{2} \bar{X}+C_{x}} \\
E\left(e_{0}\right)=E\left(e_{1}\right)=0, E\left(e_{0}{ }^{2}\right)=\left(\frac{1-f}{n}\right) \bar{Y}^{2} C_{y}{ }^{2}, E\left(e_{1}{ }^{2}\right)=\left(\frac{1-f}{n}\right) \bar{X}^{2} C_{x}{ }^{2}, E\left(e_{0} e_{1}\right)=\left(\frac{1-f}{n}\right) \rho C_{x} C_{y}
\end{gathered}
$$

Substitute the values of $e_{0}$ and $e_{1}$ in the proposed class of estimators and neglecting the higher order expressions, we get

$$
\begin{aligned}
& B\left(\hat{\bar{Y}}_{P i}\right)=E\left(\hat{\bar{Y}}_{P i}-\bar{Y}\right) \\
& B\left(\hat{\bar{Y}}_{P i}\right)=\frac{1}{2}\left[2 \bar{Y}\left(P_{i}-1\right)+P_{i}\left\{B\left(\hat{\bar{Y}}_{M R i}\right)+B\left(\hat{\bar{Y}}_{M P i}\right)\right\}+Q_{i}\left\{B\left(\hat{\bar{Y}}_{M R i}\right)-B\left(\hat{\bar{Y}}_{M P i}\right)\right\}\right]
\end{aligned}
$$

WhereB $\left(\hat{\bar{Y}}_{M R i}\right)=\delta \bar{Y}\left[\theta_{i}^{2} C_{x}^{2}-\theta_{i} \rho \mathrm{C}_{\mathrm{x}} \mathrm{C}_{\mathrm{y}}\right]$, andB $\left(\hat{\bar{Y}}_{M P i}\right)=\delta \bar{Y}\left[\theta_{i} \rho \mathrm{C}_{\mathrm{x}} \mathrm{C}_{\mathrm{y}}\right], \mathrm{i}=1,2,3,4$,

$$
\delta=\left(\frac{1-\mathrm{f}}{\mathrm{n}}\right), \theta_{1}=\frac{C_{x} \bar{X}}{C_{x} \bar{X}+\beta_{1}}, \theta_{2}=\frac{\beta_{1} \bar{X}}{\beta_{1} \bar{X}+C_{x}} \theta_{3}=\frac{C_{x} \bar{X}}{C_{x} \bar{X}+\rho}, \theta_{4}=\frac{\rho \bar{X}}{\rho \bar{X}+C_{x}}, \theta_{5}=\frac{C_{x} \bar{X}}{C_{x} \bar{X}+\beta_{2}}, \theta_{6}=\frac{\beta_{2} \bar{X}}{\beta_{2} \bar{X}+C_{x}}
$$

Where $P_{i}=\left(\alpha_{i} \lambda_{i}+\left(1-\alpha_{i}\right) \gamma_{i}\right)$ and $Q_{i}=\left(\alpha_{i} \lambda_{i}-\left(1-\alpha_{i}\right) \gamma_{i}\right)$

Where $\lambda_{i}$ and $\gamma_{i}$ are as defined above. If we assume that $a_{i}=0, b_{i}=0$ and $\alpha_{i}=1$ then the proposed estimators are exactly equal to the estimators given in Table 1 .If $a_{i}=0, b_{i}=0$ and $\alpha_{i}=0$ then the proposed estimators are exactly equal to the estimators given in Table 2. If we assume that $a_{i}=B\left(\hat{\bar{Y}}_{M R i}\right), b_{i}=B\left(\hat{\bar{Y}}_{M P i}\right), \alpha_{i}=1$ or $\alpha_{i}=0$, then the proposed estimators are almost unbiased ratio estimators corresponding to the estimators given in Table 1 and 2.

The detailed derivation of the mean squared errors are given in the appendix and the final expression is obtained with only first order approximation in the Taylor series expansion as,

$$
\begin{aligned}
& \operatorname{MSE}\left(\hat{\bar{Y}}_{P i}\right)=E\left(\hat{\bar{Y}}_{P i}-\bar{Y}\right)^{2} \\
& \operatorname{MSE}\left(\hat{\bar{Y}}_{P i}\right)=\frac{1}{4} {\left[4 \bar{Y}^{2}\left(P_{i}-1\right)^{2}+\left(P_{i}+Q_{i}\right)^{2}\left\{\operatorname{MSE}\left(\hat{\bar{Y}}_{M R i}\right)+2 \bar{Y} B\left(\hat{\bar{Y}}_{M R i}\right)\right\}\right.} \\
&+\left(P_{i}-Q_{i}\right)^{2}\left\{\operatorname{MSE}\left(\hat{\bar{Y}}_{M P i}\right)+2 \bar{Y} B\left(\hat{\bar{Y}}_{M P i}\right)\right\} \\
&\left.-4 \bar{Y}\left\{\left(P_{i}+Q_{i}\right) B\left(\hat{\bar{Y}}_{M R i}\right)+\left(P_{i}-Q_{i}\right) B\left(\hat{\bar{Y}}_{M P i}\right)\right\}+2\left(P_{i}{ }^{2}-Q_{i}{ }^{2}\right) \mathrm{V}\left(\bar{y}_{S r s}\right)\right]
\end{aligned}
$$

WhereB $\left(\hat{\bar{Y}}_{M R i}\right)=\delta \bar{Y}\left[\theta_{i}{ }^{2} C_{x}{ }^{2}-\theta_{i} \rho \mathrm{C}_{\mathrm{x}} \mathrm{C}_{\mathrm{y}}\right], \quad \mathrm{B}\left(\hat{\bar{Y}}_{M P i}\right)=\delta \bar{Y}\left[\theta_{i} \rho \mathrm{C}_{\mathrm{x}} \mathrm{C}_{\mathrm{y}}\right]$

$$
\operatorname{MSE}\left(\hat{\bar{Y}}_{M R i}\right)=\delta \bar{Y}^{2}\left[C_{y}{ }^{2}+\theta_{i}{ }^{2} C_{x}{ }^{2}-2 \theta_{i} \rho \mathrm{C}_{\mathrm{x}} \mathrm{C}_{\mathrm{y}}\right]
$$

$\operatorname{MSE}\left(\hat{\bar{Y}}_{M P i}\right)=\delta \bar{Y}^{2}\left[C_{y}{ }^{2}+\theta_{i}{ }^{2} C_{x}{ }^{2}+2 \theta_{i} \rho \mathrm{C}_{\mathrm{x}} \mathrm{C}_{\mathrm{y}}\right]$ andV $\left(\bar{y}_{s r s}\right)=\delta S_{y}{ }^{2}=\delta \bar{Y}^{2} C_{y}{ }^{2}$

The optimal value of $\alpha_{i}$ 's are determined by minimizing the MSE $\left(\hat{\bar{Y}}_{p i}\right)$ with respect to $\alpha_{i}$. For this differentiate MSE with respect to $\alpha_{i}$ and equate to zero.

$\frac{\partial M S E}{\partial \alpha_{i}}=0$, and we get the value of $\alpha_{i}$, as 


$$
\alpha_{i}=\frac{\bar{Y}^{2}\left(\gamma_{i}-1\right)\left(\gamma_{i}-\lambda_{i}\right)+\gamma_{i}^{2}\left\{M S E\left(\hat{\bar{Y}}_{M P i}\right)+2 \bar{Y} B\left(\hat{\bar{Y}}_{M P i}\right)\right\}+\bar{Y}\left\{\lambda_{i} B\left(\hat{\bar{Y}}_{M R i}\right)-\gamma_{i} B\left(\hat{\bar{Y}}_{M P i}\right)\right\}}{-\lambda_{i} \gamma_{i} \mathrm{~V}\left(\bar{y}_{S r S}\right)}
$$

Where $\lambda_{i}=\frac{S_{y}}{S_{y}+a_{i} C_{y}}, \gamma_{i}=\frac{S_{y}}{S_{y}+b_{i} C_{y}}, \mathrm{i}=1,2,3,4,5,6$

\section{Efficiency Comparison}

If that $a_{i}=B\left(\hat{\bar{Y}}_{M R i}\right), b_{i}=B\left(\hat{\bar{Y}}_{M R i}\right)$ then $\lambda_{i}=\frac{S_{y}}{S_{y}+B\left(\hat{Y}_{M R i}\right) C_{y}}, \gamma_{i}=\frac{S_{y}}{s_{y}+B\left(\hat{Y}_{M P i}\right) C_{y}}, \mathrm{i}=1,2,3,4,5,6$.

Substitute these values in the biased estimator and taking expectation we get the proposed estimators are almost unbiased.The efficiency comparison of the mean squared errorsof proposed estimatorsunder optimum conditions with that of the existing estimators are as follows

3.1 Comparison of Proposed estimators and Modified Ratio Estimator

$$
\begin{gathered}
\operatorname{MSE}\left(\hat{\bar{Y}}_{M R i}\right) \geq \operatorname{MSE}\left(\hat{\bar{Y}}_{P i}\right) \\
\delta \bar{Y}^{2}\left[C_{y}{ }^{2}+\theta_{i}{ }^{2} C_{x}{ }^{2}-2 \theta_{i} \rho C_{\mathrm{x}} \mathrm{C}_{\mathrm{y}}\right] \\
\geq \bar{Y}^{2}\left(\alpha_{i} \lambda_{i}+\left(1-\alpha_{i}\right) \gamma_{j}-1\right)^{2}+\alpha_{i}{ }^{2} \lambda_{i}{ }^{2}\left\{M S E\left(\hat{\bar{Y}}_{M R i}\right)+2 \bar{Y} B\left(\hat{\bar{Y}}_{M R i}\right)\right\} \\
+\left(1-\alpha_{i}\right)^{2} \gamma_{i}{ }^{2}\left\{M S E\left(\hat{\bar{Y}}_{M P i}\right)+2 \bar{Y} B\left(\hat{\bar{Y}}_{M P i}\right)\right\} \\
-2 \bar{Y}\left\{\alpha_{i} \lambda_{i} B\left(\hat{\bar{Y}}_{M R i}\right)+\left(1-\alpha_{i}\right) \gamma_{i} B\left(\hat{\bar{Y}}_{M P i}\right)\right\}+2\left(\alpha_{i} \lambda_{i}\left(1-\alpha_{i}\right) \gamma_{i} \mathrm{~V}\left(\bar{y}_{s r s}\right)\right)
\end{gathered}
$$

$$
\begin{aligned}
& \delta \bar{Y}^{2}\left[{C_{y}}^{2}+\theta_{i}^{2} C_{x}^{2}-2 \theta_{i} \rho \mathrm{C}_{\mathrm{x}} \mathrm{C}_{\mathrm{y}}\right] \\
& \geq \bar{Y}^{2}\left(\alpha_{i} \lambda_{i}+\left(1-\alpha_{i}\right) \gamma_{i}-1\right)^{2} \\
& +\delta \bar{Y}^{2}\left(C_{y}{ }^{2}\left(\alpha_{i} \lambda_{i}+\left(1-\alpha_{i}\right) \gamma_{i}\right)^{2}+\theta^{2} C_{x}{ }^{2}\left(3 \alpha_{i}{ }^{2} \lambda_{i}{ }^{2}+\left(1-\alpha_{i}\right)^{2} \gamma_{i}{ }^{2}-2 \alpha_{i} \lambda_{i}\right)\right. \\
& \left.+2 \theta \rho C_{x} C_{y}\left(\alpha_{i} \lambda_{i}-\left(1-\alpha_{i}\right) \gamma_{i}\right)-2\left(\alpha_{i}{ }^{2} \lambda_{i}^{2}-\left(1-\alpha_{i}\right)^{2} \gamma_{i}{ }^{2}\right)\right) \\
& \delta \bar{Y}^{2}\left[C_{y}{ }^{2}+\theta^{2} C_{x}{ }^{2}-2 \theta \rho C_{x} C_{y}\right] \geq \bar{Y}^{2}\left(P_{i}-1\right)^{2} \\
& +\delta \bar{Y}^{2}\left\{P_{i}{ }^{2} C_{y}{ }^{2}+\theta^{2} C_{x}{ }^{2}\left(P_{i}{ }^{2}+\left(P_{i}+Q_{i}\right)\left(Q_{i}-1\right)-2 \theta \rho C_{x} C_{y} Q_{i}\left(2 P_{i}-1\right)\right)\right\}
\end{aligned}
$$

$$
\begin{gathered}
2 \delta \theta \rho C_{x} C_{y}\left(Q_{i}\left(2 P_{i}-1\right)-1\right) \\
\geq\left(P_{i}-1\right)^{2}+\delta\left\{C_{y}{ }^{2}\left(P_{i}{ }^{2}-1\right)+\theta^{2} C_{x}{ }^{2}\left(P_{i}{ }^{2}+\left(P_{i}+Q_{i}\right)\left(Q_{i}-1\right)-1\right)\right\} \\
\rho \geq \frac{\left(P_{i}-1\right)^{2}+\delta\left[C_{y}^{2}\left(P_{i}{ }^{2}-1\right)+\theta^{2} C_{x}{ }^{2}\left(P_{i}{ }^{2}+\left(P_{i}+Q_{i}\right)\left(P_{i}-1\right)-1\right)\right]}{2 \delta \theta C_{x} C_{y}\left(Q_{i}\left(2 P_{i}-1\right)-1\right)}
\end{gathered}
$$

\subsection{Comparison of Proposed estimators and Modified Product Estimators}

$$
\operatorname{MSE}\left(\hat{\bar{Y}}_{M P i}\right) \geq \operatorname{MSE}\left(\hat{\bar{Y}}_{P i}\right)
$$




$$
\begin{aligned}
\delta \bar{Y}^{2}\left[{C_{y}}^{2}+\theta_{i}{ }^{2}\right. & \left.C_{x}{ }^{2}+2 \theta_{i} \rho \mathrm{C}_{\mathrm{x}} \mathrm{C}_{\mathrm{y}}\right] \\
& \geq \bar{Y}^{2}\left(\alpha_{i} \lambda_{i}+\left(1-\alpha_{i}\right) \gamma_{j}-1\right)^{2}+\alpha_{i}{ }^{2} \lambda_{i}{ }^{2}\left\{\operatorname{MSE}\left(\hat{\bar{Y}}_{M R i}\right)+2 \bar{Y} B\left(\hat{\bar{Y}}_{M R i}\right)\right\} \\
& +\left(1-\alpha_{i}\right)^{2} \gamma_{i}{ }^{2}\left\{M S E\left(\hat{\bar{Y}}_{M P i}\right)+2 \bar{Y} B\left(\hat{\bar{Y}}_{M P i}\right)\right\} \\
& -2 \bar{Y}\left\{\alpha_{i} \lambda_{i} B\left(\hat{\bar{Y}}_{M R i}\right)+\left(1-\alpha_{i}\right) \gamma_{i} B\left(\hat{\bar{Y}}_{M P i}\right)\right\}+2\left(\alpha_{i} \lambda_{i}\left(1-\alpha_{i}\right) \gamma_{i} \mathrm{~V}\left(\bar{y}_{s r s}\right)\right)
\end{aligned}
$$

$$
\begin{aligned}
\delta \bar{Y}^{2}\left[C_{y}{ }^{2}+\theta_{i}{ }^{2} C_{x}{ }^{2}+2 \theta_{i} \rho C_{\mathrm{x}} \mathrm{C}_{\mathrm{y}}\right] & \\
\geq & \bar{Y}^{2}\left(\alpha_{i} \lambda_{i}+\left(1-\alpha_{i}\right) \gamma_{i}-1\right)^{2} \\
& +\delta \bar{Y}^{2}\left(C_{y}{ }^{2}\left(\alpha_{i} \lambda_{i}+\left(1-\alpha_{i}\right) \gamma_{i}\right)^{2}+\theta^{2} C_{x}{ }^{2}\left(3 \alpha_{i}{ }^{2} \lambda_{i}{ }^{2}+\left(1-\alpha_{i}\right)^{2} \gamma_{i}{ }^{2}-2 \alpha_{i} \lambda_{i}\right)\right. \\
& \left.+2 \theta \rho C_{x} C_{y}\left(\alpha_{i} \lambda_{i}-\left(1-\alpha_{i}\right) \gamma_{i}\right)-2\left(\alpha_{i}{ }^{2} \lambda_{i}{ }^{2}-\left(1-\alpha_{i}\right)^{2} \gamma_{i}{ }^{2}\right)\right)
\end{aligned}
$$

$$
\begin{gathered}
2 \delta \theta \rho C_{x} C_{y}\left(Q_{i}\left(2 P_{i}-1\right)+1\right) \\
\geq\left(P_{i}-1\right)^{2}+\delta\left\{C_{y}{ }^{2}\left(P_{i}{ }^{2}-1\right)+\theta^{2} C_{x}{ }^{2}\left(P_{i}^{2}+\left(P_{i}+Q_{i}\right)\left(Q_{i}-1\right)-1\right)\right\} \\
\rho \geq \frac{\left(P_{i}-1\right)^{2}+\delta\left\{C_{y}^{2}\left(P_{i}{ }^{2}-1\right)+\theta^{2} C_{x}{ }^{2}\left(P_{i}{ }^{2}-\left(P_{i}+Q_{i}\right)\left(Q_{i}-1\right)-1\right)\right.}{2 \delta \theta C_{x y}\left(Q_{i}\left(2 P_{i}-1\right)+1\right)}
\end{gathered}
$$

\section{Numerical Study}

In this section we consider three natural populations,population 1 (Singh and Chaudhary [11] page 177),population 2 (Khoshnevisan et.al.[6])and Population 3 (Cochran[2] page 152) and are used to obtain the biases and mean squared errors and also used to compare the percentage relative efficiency of proposed estimator with that of the existing estimators.The computed values of constants and parameters of these populations are given below

Table 3 : Parameters and Constants of Different Populations

\begin{tabular}{|c|c|c|c|}
\hline Constants & Population 1 & Population 2 & Population 3 \\
\hline $\mathrm{N}$ & 34.000 & 20.000 & 49.000 \\
\hline $\mathrm{n}$ & 5.000 & 5.000 & 5.000 \\
\hline $\bar{Y}$ & 199.441 & 19.550 & 127.796 \\
\hline $\bar{X}$ & 856.412 & 18.800 & 103.143 \\
\hline$S_{x}$ & 733.141 & 7.413 & 104.405 \\
\hline$S_{y}$ & 150.215 & 6.944 & 123.121 \\
\hline$C_{x}$ & 0.856 & 0.394 & 1.012 \\
\hline$C_{y}$ & 0.753 & 0.355 & 0.963 \\
\hline$\beta_{1}$ & 7.955 & 3.061 & 4.777 \\
\hline$\beta_{2}$ & 13.367 & 0.547 & 7.511 \\
\hline$\rho$ & 0.445 & -0.919 & 0.982 \\
\hline$\theta_{1}$ & 0.989 & 0.708 & 0.956 \\
\hline
\end{tabular}




\begin{tabular}{|c|c|c|c|}
\hline$\theta_{2}$ & 1.000 & 0.993 & 0.998 \\
\hline$\theta_{3}$ & 0.856 & 0.415 & 1.003 \\
\hline$\theta_{4}$ & 0.998 & 1.023 & 0.990 \\
\hline$\theta_{5}$ & 0.843 & 0.383 & 0.944 \\
\hline$\theta_{6}$ & 1.000 & 0.963 & 0.999 \\
\hline$\lambda_{1}$ & 0.931 & 0.975 & 0.996 \\
\hline$\lambda_{2}$ & 0.929 & 0.960 & 0.988 \\
\hline$\lambda_{3}$ & 0.953 & 0.988 & 0.988 \\
\hline$\lambda_{4}$ & 0.930 & 0.958 & 0.990 \\
\hline$\lambda_{5}$ & 0.955 & 0.989 & 0.998 \\
\hline$\lambda_{6}$ & 0.929 & 0.961 & 0.988 \\
\hline$\gamma_{1}$ & 0.954 & 1.014 & 0.859 \\
\hline$\gamma_{2}$ & 0.953 & 1.020 & 0.854 \\
\hline$\gamma_{3}$ & 0.960 & 1.008 & 0.853 \\
\hline$\gamma_{4}$ & 0.953 & 1.020 & 0.855 \\
\hline$\gamma_{5}$ & 0.960 & 1.007 & 0.860 \\
\hline$\gamma_{6}$ & 0.953 & 1.019 & 0.853 \\
\hline$\alpha_{1}$ & 0.710 & -0.102 & 0.983 \\
\hline$\alpha_{2}$ & 0.709 & 0.079 & 0.958 \\
\hline$\alpha_{3}$ & 0.733 & -0.535 & 0.955 \\
\hline$\alpha_{4}$ & 0.709 & 0.093 & 0.962 \\
\hline$\alpha_{5}$ & 0.736 & -0.620 & 0.992 \\
\hline$\alpha_{6}$ & 0.709 & 0.065 & 0.957 \\
\hline & & & \\
\hline
\end{tabular}


New Class of Almost Unbiased Modified Ratio Cum Product Estimators with Knownparameters of Auxiliary Variables

Table 4:Bias and MSE of Proposed Estimators

\begin{tabular}{|c|r|r|r|r|r|r|}
\hline \multirow{2}{*}{$\begin{array}{c}\text { Proposed } \\
\text { Estimators }\end{array}$} & \multicolumn{2}{|c|}{ Population 1 } & \multicolumn{2}{c|}{ Population 2 } & \multicolumn{2}{c|}{ Population 3 } \\
\cline { 2 - 7 } & \multicolumn{1}{c|}{ Bias } & \multicolumn{1}{c|}{ MSE } & \multicolumn{1}{c|}{ Bias } & \multicolumn{1}{c|}{ MSE } & \multicolumn{1}{c|}{ MSE } \\
\hline$\hat{\bar{Y}}_{p 1}$ & $1.78 \mathrm{E}-15$ & 2560.326 & $3.01 \mathrm{E}-06$ & 1.033955 & $9.88 \mathrm{E}-15$ & 96.78642 \\
\hline$\hat{\bar{Y}}_{p 2}$ & $7.11 \mathrm{E}-15$ & 2545.273 & $2.50 \mathrm{E}-06$ & 1.063967 & $4.44 \mathrm{E}-15$ & 90.55698 \\
\hline$\hat{\bar{Y}}_{p 3}$ & $4.26 \mathrm{E}-14$ & 2730.305 & $3.17 \mathrm{E}-06$ & 1.022477 & $5.33 \mathrm{E}-15$ & 89.59797 \\
\hline$\hat{\bar{Y}}_{p 4}$ & $-1.60 \mathrm{E}-14$ & 2548.292 & $2.43 \mathrm{E}-06$ & 1.067739 & $-2.18 \mathrm{E}-14$ & 92.03092 \\
\hline$\hat{\bar{Y}}_{p 5}$ & $-1.07 \mathrm{E}-14$ & 2744.652 & $3.16 \mathrm{E}-06$ & 1.022694 & $1.61 \mathrm{E}-14$ & 97.90284 \\
\hline$\hat{\bar{Y}}_{p 6}$ & $-1.78 \mathrm{E}-15$ & 2545.201 & $2.57 \mathrm{E}-06$ & 1.060262 & $-1.55 \mathrm{E}-14$ & 90.40969 \\
\hline
\end{tabular}

Table 5:Bias and MSE of Existing Estimators

\begin{tabular}{|c|r|r|r|r|r|r|}
\hline \multirow{2}{*}{$\begin{array}{c}\text { Existing } \\
\text { Estimators }\end{array}$} & \multicolumn{2}{|c|}{ Population 1 } & \multicolumn{2}{c|}{ Population 2 } & \multicolumn{2}{c|}{ Population 3 } \\
\cline { 2 - 7 } & \multicolumn{1}{|c|}{ Bias } & \multicolumn{1}{l|}{ MSE } & Bias & \multicolumn{1}{c|}{ MSE } & \multicolumn{1}{c|}{ Bias } & \multicolumn{1}{c|}{ MSE } \\
\hline$\hat{\bar{Y}}_{M R 1}$ & 14.737 & 4860.970 & 0.496 & 22.153 & 0.491 & 99.939 \\
\hline$\hat{\bar{Y}}_{M R 2}$ & 15.159 & 4924.565 & 0.825 & 30.698 & 1.491 & 110.643 \\
\hline$\hat{\bar{Y}}_{M R 3}$ & 9.895 & 4155.649 & 0.235 & 14.890 & 1.610 & 112.522 \\
\hline$\hat{\bar{Y}}_{M R 4}$ & 15.075 & 4911.803 & 0.864 & 31.684 & 1.297 & 107.830 \\
\hline$\hat{\bar{Y}}_{M R 5}$ & 9.480 & 4097.819 & 0.212 & 14.202 & 0.203 & 98.754 \\
\hline$\hat{\bar{Y}}_{M R 6}$ & 15.161 & 4924.872 & 0.787 & 29.728 & 1.510 & 110.929 \\
\hline$\hat{\bar{Y}}_{M P 1}$ & 9.664 & 12570.470 & -0.267 & 1.243 & 21.012 & 10840.980 \\
\hline$\hat{\bar{Y}}_{M P 2}$ & 9.768 & 12716.740 & -0.375 & 1.354 & 21.928 & 11320.110 \\
\hline$\hat{\bar{Y}}_{M P 3}$ & 8.358 & 10823.600 & -0.157 & 2.641 & 22.033 & 11375.270 \\
\hline$\hat{\bar{Y}}_{M p 4}$ & 9.747 & 12687.500 & -0.387 & 1.450 & 21.756 & 11229.150 \\
\hline$\hat{\bar{Y}}_{M P 5}$ & 8.234 & 10666.710 & -0.145 & 2.882 & 20.733 & 10696.890 \\
\hline$\hat{\bar{Y}}_{M p 6}$ & 9.768 & 12717.440 & -0.364 & 1.273 & 21.945 & 11328.770 \\
\hline
\end{tabular}

Table 6: Percentage Relative Efficiency of Proposed Estimators

\begin{tabular}{|c|c|c|c|c|c|c|}
\hline \multirow{2}{*}{$\begin{array}{c}\text { Proposed } \\
\text { Estimators }\end{array}$} & \multicolumn{2}{|c|}{ Population 1 } & \multicolumn{2}{c|}{ Population 2 } & \multicolumn{2}{c|}{ Population 3 } \\
\cline { 2 - 7 } & $\begin{array}{c}\text { Modified } \\
\text { Ratio }\end{array}$ & $\begin{array}{c}\text { Modified } \\
\text { Product }\end{array}$ & $\begin{array}{c}\text { Modified } \\
\text { Ratio }\end{array}$ & $\begin{array}{c}\text { Modified } \\
\text { Product }\end{array}$ & $\begin{array}{c}\text { Modified } \\
\text { Ratio }\end{array}$ & $\begin{array}{c}\text { Modified } \\
\text { Product }\end{array}$ \\
\hline$\hat{\bar{Y}}_{p 1}$ & 189.857 & 490.971 & 2142.513 & 120.194 & 103.257 & 11200.930 \\
\hline$\hat{\bar{Y}}_{p 2}$ & 193.479 & 499.622 & 2885.204 & 127.221 & 122.180 & 12500.540 \\
\hline$\hat{\bar{Y}}_{p 3}$ & 152.205 & 396.424 & 1456.240 & 258.270 & 125.586 & 12695.900 \\
\hline$\hat{\bar{Y}}_{p 4}$ & 192.749 & 497.883 & 2967.437 & 135.808 & 117.167 & 12201.500 \\
\hline$\hat{\bar{Y}}_{p 5}$ & 149.302 & 388.636 & 1388.658 & 281.770 & 100.870 & 10926.030 \\
\hline$\hat{\bar{Y}}_{p 6}$ & 193.496 & 499.664 & 2803.839 & 120.101 & 122.696 & 12530.480 \\
\hline
\end{tabular}




\section{Conclusions}

In this paper we have suggested a new class of the modified ratio cum product estimators for finite population mean of the study variable $\mathrm{Y}$ with known parameters of the auxiliary variable. The biases and mean squared errors of the proposed estimators are obtained and compared with that of some existing modified ratio and modified product estimators.Theoretically we have shown that the proposed estimator is always more efficient than other existing estimators under the optimum values of $\alpha_{\mathrm{i}}$ and $a_{i}=B\left(\hat{\bar{Y}}_{M R i}\right), b_{i}=B\left(\hat{\bar{Y}}_{M R i}\right)$. We have also studied the performance of the proposed estimators for certain known natural populations, it shows that the proposed estimator has less bias and mean squared error than all these existing estimators. That is the proposed estimator is more efficient than all these existing estimators. Hence we strongly recommended that the proposed estimator is more preferable than these existing estimators.

\section{References}

1. Cochran W.G.(1940):The estimation of the yields of the cereal experiments by sampling for the ratio of grain to total produce, The Journal of Agricultural Science, 30, 262-275.

2. Cochran, W. G. (1977):Sampling Techniques. Third Edition, Wiley Eastern Limited.

3. Jambulingam Subramani and Master Ajith (2016):Improved Ratio cum Product Estimator with Known Coefficient of Variation inSimple Random SamplingJ. Adv. Res. Appl. Math. Stat.; 1(2), 60-70

4. Jambulingam Subramani and Master Ajith (2016):Modified Ratio cum Product Estimators for Estimation of Finite Population Mean with Known Correlation Coefficient Biom. Biostat. Int J , 4(6): 00113

5. Khoshnevisan M., Singh R., Chauhan P., Sawan N. and Smarandache F. (2007): A general family of estimators for estimating population mean using known value of some population parameter(s), Far East Journal of Theoretical Statistics 22, 181-191

6. Murthy, M.N. (1964): Product method of estimation. Sankhya A, 26, 69-74.

7. Murthy, M.N. (1967): Sampling theory and methods. Statistical Publishing Society, Calcutta, India

8. Rajesh Tailor and Balkishan Sharma (2009): A Modified Ratio-cum-product estimator of finite population mean using known coefficient of variation and coefficient of kurtosis statistics in transition, July Vol. 10, No. 1, pp. 15-24

9. Ramkrishna S. Solanki, Housila P. Singh and Surya K. Pal (2015): Improved ratio-type estimators of finite population variance using quartiles, Hacettepe Journal of Mathematics and Statistics Volume 44 (3) $747-754$

10. Singh, D. and Chaudhary, F.S. (1986):Theory and analysis of sample survey designs. New Age International Publisher

11. Singh, H. P. and Agnihotri, N.(2008): A general procedure of estimating population mean using auxiliary information in sample surveys. Statistics in Transition- new series, 9, 7187

12. Singh, H.P., Tailor, R., Tailor, R. and Kakran, M.S.(2004):Animproved estimator of population mean using power transformation. Journal of the Indian Society of Agricultural Statistics, 58(2), 223-230, 
13. Sisodia B.V.S. and V.K. Dwivedi (1981): "A modified ratio estimator using coefficient of variation of auxiliary variable”, Jour. Ind. Soc. Agri. Stat., Vol. 33(1), Pp. 13-18,

14. Subramani, J (2013): "Generalized modified ratio estimator for estimation of finite population mean," Journal of Modern Applied Statistical Methods, vol.12, pp.121-155.

15. Subramani J.and G. Kumarapandiyan (2012): Modified Ratio Estimators for Population Mean Using Function of Quartiles of Auxiliary Variable, Bonfring International Journal of Industrial Engineering and Management Science, Vol. 2, No. 2,

16. Subramani, J.,and Kumarapandiyan, (2012) G. Variance estimation using quartiles and their functionsof an auxiliary variable, International Journal of Statistics and Applications $2(5), 67-72$.

17. Subramani and G.Kumarapandiyan (2012): A class of almost unbiased modified ratio estimators for population mean withknown population parameters;Elixir Statistics 44 7411-7415

18. Upadhyaya, L.N. and Singh, H.P(1999): Use of transformed auxiliary variable in estimating the finite population mean, Biometrical Journal 41 (5), 627-636,

19. Yan, Z. and Tian, B. (2010): Ratio Method to the Mean Estimation Using Co-efficient of Skewness of Auxiliary Variable, ICICA 2010, Part II, CCIS 106, pp. 103-110 


\section{APPENDIX}

\section{Bias and MSE of Proposed Estimators} variables are

The proposed class of ratio cum product estimators with known parameters of auxiliary

$$
\begin{gathered}
\widehat{\bar{Y}}_{P 1}=\alpha_{1} \lambda_{1} \bar{y}\left(\frac{C_{x} \bar{X}+\beta_{1}}{C_{x} \bar{x}+\beta_{1}}\right)+\left(1-\alpha_{1}\right) \gamma_{1} \bar{y}\left(\frac{C_{x} \bar{x}+\beta_{1}}{C_{x} \bar{X}+\beta_{1}}\right) \\
\widehat{Y}_{P 2}=\alpha_{2} \lambda_{2} \bar{y}\left(\frac{\beta_{1} \bar{X}+C_{x}}{\beta_{1} \bar{x}+C_{x}}\right)+\left(1-\alpha_{2}\right) \gamma_{2} \bar{y}\left(\frac{\beta_{1} \bar{x}+C_{x}}{\beta_{1} \bar{X}+C_{x}}\right) \\
\widehat{\bar{Y}}_{P 3}=\alpha_{3} \lambda_{3} \bar{y}\left(\frac{C_{x} \bar{X}+\rho}{C_{x} \bar{x}+\rho}\right)+\left(1-\alpha_{3}\right) \gamma_{3} \bar{y}\left(\frac{C_{x} \bar{x}+Q_{r}}{C_{x} \bar{X}+Q_{r}}\right) \\
\widehat{\bar{Y}}_{P 4}=\alpha_{4} \lambda_{4} \bar{y}\left(\frac{\rho \bar{X}+C_{x}}{\rho \bar{x}+C_{x}}\right)+\left(1-\alpha_{4}\right) \gamma_{4} \bar{y}\left(\frac{\rho \bar{x}+C_{x}}{\rho \bar{X}+C_{x}}\right) \\
\widehat{\bar{Y}}_{P 5}=\alpha_{5} \lambda_{5} \bar{y}\left(\frac{C_{x} \bar{X}+\beta_{2}}{C_{x} \bar{x}+\beta_{2}}\right)+\left(1-\alpha_{5}\right) \gamma_{5} \bar{y}\left(\frac{C_{x} \bar{x}+\beta_{2}}{C_{x} \bar{X}+\beta_{2}}\right) \\
\widehat{Y}_{P 6}=\alpha_{6} \lambda_{6} \bar{y}\left(\frac{\beta_{2} \bar{X}+C_{x}}{\beta_{2} \bar{x}+C_{x}}\right)+\left(1-\alpha_{6}\right) \gamma_{6} \bar{y}\left(\frac{\beta_{2} \bar{x}+C_{x}}{\beta_{2} \bar{X}+C_{x}}\right)
\end{gathered}
$$

Where $\lambda_{i}=\frac{S_{y}}{S_{y}+a_{i} C_{y}} \gamma_{i}=\frac{S_{y}}{S_{y}+b_{i} C_{y}}, \quad \mathrm{i}=1,2,3,4,5,6$

To obtain the bias and mean squared error of the proposed estimators,

Consider, $e_{0}=\frac{\bar{y}-\bar{Y}}{\bar{Y}}, e_{1}=\frac{\bar{x}-\bar{X}}{\bar{X}}, \delta=\left(\frac{1-f}{n}\right), f=\frac{n}{N}$

$\theta_{1}=\frac{C_{x} \bar{X}}{C_{x} \bar{X}+\beta_{1}}, \theta_{2}=\frac{\beta_{1} \bar{X}}{\beta_{1} \bar{X}+C_{x}} \theta_{3}=\frac{C_{x} \bar{X}}{C_{x} \bar{X}+\rho}, \theta_{4}=\frac{\rho \bar{X}}{\rho \bar{X}+C_{x}}, \theta_{5}=\frac{C_{x} \bar{X}}{C_{x} \bar{X}+\beta_{2}}, \theta_{6}=\frac{\beta_{2} \bar{X}}{\beta_{2} \bar{X}+C_{x}}$

Where $\mathrm{i}=1,2,3,4,5,6$

$$
\begin{gathered}
E\left(e_{0}\right)=E\left(e_{1}\right)=0, E\left(e_{0}{ }^{2}\right)=\delta \bar{Y}^{2} C_{y}{ }^{2}, E\left(e_{1}{ }^{2}\right)=\delta \bar{X}^{2} C_{x}{ }^{2}, \\
E\left(e_{0} e_{1}\right)=\delta \rho C_{x} C_{y}
\end{gathered}
$$

Substitute these values in $\widehat{Y}_{p i}$ and neglecting the higher order expressions, we get

$$
\begin{aligned}
& \widehat{\bar{Y}}_{p i}= \alpha_{i} \lambda_{i} \bar{Y}\left(1+e_{0}\right)\left(1+\theta_{i} e_{1}\right)^{-1}+\left(1-\alpha_{i}\right) \gamma_{i} \bar{Y}\left(1+e_{0}\right)\left(1+\theta_{i} e_{1}\right) \\
&= \bar{Y}\left\{\alpha_{i} \lambda_{i}\left(1+e_{0}\right)\left(1-\theta_{i} e_{1}+\theta_{i}{ }^{2} e_{1}{ }^{2}\right)+\left(1-\alpha_{i}\right) \gamma_{i}\left(1+e_{0}\right)\left(1+\theta_{i} e_{1}\right)\right\} \\
&= \bar{Y}\left\{\alpha_{i} \lambda_{i}\left(1-\theta_{i} e_{1}+\theta_{i}{ }^{2} e_{1}{ }^{2}+e_{0}-\theta_{i} e_{1} e_{0}\right)+\left(1-\alpha_{i}\right) \gamma_{i}\left(1+\theta_{i} e_{1}+e_{0}+\theta_{i} e_{0} e_{1}\right)\right\} \\
& \widehat{\bar{Y}}_{p i}-\bar{Y}=\bar{Y}\left\{\alpha_{i} \lambda_{i}\left(1-\theta_{i} e_{1}+\theta_{i}{ }^{2} e_{1}{ }^{2}+e_{0}-\theta_{i} e_{1} e_{0}\right)+\left(1-\alpha_{i}\right) \gamma_{i}\left(1+\theta_{i} e_{1}+e_{0}+\theta_{i} e_{0} e_{1}\right)\right\} \\
& B\left(\hat{\bar{Y}}_{P i}\right)=E\left(\hat{\bar{Y}}_{P i}-\bar{Y}\right) \\
&= E\left\{\overline { Y } \left(\alpha_{i} \lambda_{i}\left(1-\theta_{i} e_{1}+\theta_{i}{ }^{2} e_{1}{ }^{2}+e_{0}-\theta_{i} e_{1} e_{0}\right)+\left(1-\alpha_{i}\right) \gamma_{i}\left(1+\theta_{i} e_{1}+e_{0}+\right.\right.\right. \\
&\left.\left.\left.\theta_{i} e_{0} e_{1}\right)-1\right)\right\} \\
&= \delta \bar{Y}\left\{\alpha_{i} \lambda_{i}\left(1+\theta_{i}{ }^{2} C_{x}{ }^{2}-\theta_{i} \rho C_{x} C_{y}\right)+\left(1-\alpha_{i}\right) \gamma_{i}\left(1+\theta_{i} \rho C_{x} C_{y}\right)-1\right\} \\
&
\end{aligned}
$$


$B\left(\hat{\bar{Y}}_{P i}\right)=\bar{Y}\left(\alpha_{i} \lambda_{i}+\left(1-\alpha_{i}\right) \gamma_{i}-1\right\}+\alpha_{i} \lambda_{i} B\left(\hat{\bar{Y}}_{M R i}\right)+\left(1-\alpha_{i}\right) \gamma_{i} B\left(\hat{\bar{Y}}_{M P i}\right)$

Take a substitution $P_{i}=\left(\alpha_{i} \lambda_{i}+\left(1-\alpha_{i}\right) \gamma_{i}\right)$ and $Q_{i}=\left(\alpha_{i} \lambda_{i}-\left(1-\alpha_{i}\right) \gamma_{i}\right)$ then

$B\left(\hat{\bar{Y}}_{P i}\right)=\bar{Y}\left(P_{i}-1\right\}+\frac{\left(P_{i}+Q_{i}\right)}{2} B\left(\hat{\bar{Y}}_{M R i}\right)+\frac{\left(P_{i}-Q_{i}\right)}{2} B\left(\hat{\bar{Y}}_{M P i}\right)$
$B\left(\hat{\bar{Y}}_{P i}\right)=\frac{1}{2}\left[2 \bar{Y}\left(P_{i}-1\right)+P_{i}\left\{B\left(\hat{\bar{Y}}_{M R i}\right)+B\left(\hat{\bar{Y}}_{M P i}\right)\right\}+Q_{i}\left\{B\left(\hat{\bar{Y}}_{M R i}\right)-B\left(\hat{\bar{Y}}_{M P i}\right)\right\}\right]$

The mean squared errors of the proposed estimatorsare

$$
\begin{aligned}
& \operatorname{MSE}\left(\hat{\bar{Y}}_{P i}\right)=E\left(\hat{\bar{Y}}_{P i}-\bar{Y}\right)^{2} \\
& =E\left\{\bar{Y}\left(\alpha_{i} \lambda_{i}\left(1-\theta_{i} e_{1}+\theta^{2} e_{1}^{2}+e_{0}-\theta_{i} e_{1} e_{0}\right)+\left(1-\alpha_{i}\right) \gamma_{i}\left(1+\theta_{i} e_{1}+e_{0}+\theta_{i} e_{0} e_{1}\right)-1\right)^{2}\right\} \\
& =E\left\{\overline { Y } ^ { 2 } \left(\alpha_{i}{ }^{2} \lambda_{i}{ }^{2}\left(1-\theta_{i} e_{1}+\theta_{i}{ }^{2} e_{1}{ }^{2}+e_{0}-\theta_{i} e_{1} e_{0}\right)^{2}+\left(1-\alpha_{i}\right)^{2} \gamma_{i}{ }^{2}\left(1+\theta_{i} e_{1}+\theta_{i} e_{0} e_{1}\right)^{2}+1\right.\right. \\
& +2 \alpha_{i} \lambda_{i}\left(1-\alpha_{i}\right) \gamma_{i}\left(1-\theta_{i} e_{1}+\theta_{i}^{2} e_{1}^{2}+e_{0}-\theta_{i} e_{1} e_{0}\right)\left(1+\theta_{i} e_{1}+e_{0}+\theta_{i} e_{0} e_{1}\right) \\
& -2 \alpha_{i} \lambda_{i}\left(1-\theta e_{1}+\theta_{i}^{2} e_{1}^{2}+e_{0}-\theta_{i} e_{1} e_{0}\right)-2(1 \\
& \left.\left.-\alpha_{i}\right) \gamma_{i}\left(1+\theta_{i} e_{1}+e_{0}+\theta_{i} e_{0} e_{1}\right)\right\} \\
& =\delta \bar{Y}^{2}\left\{\alpha_{i}^{2} \lambda_{i}^{2}\left(1+3 \theta_{i}^{2} C_{x}^{2}+C_{y}^{2}-4 \theta_{i} \rho C_{x} C_{y}\right)\right. \\
& +\left(1-\alpha_{i}\right)^{2} \gamma_{i}^{2}\left(1+\theta_{i} \theta^{2} C_{x}{ }^{2}+C_{y}{ }^{2}+4 \theta_{i} \rho C_{x} C_{y}\right)+2 \alpha_{i} \lambda_{i}\left(1-\alpha_{i}\right) \gamma_{i}\left(1+C_{y}{ }^{2}\right) \\
& \left.-2 \alpha_{i} \lambda_{i}\left(1+\theta_{i}{ }^{2} C_{x}^{2}-\theta_{i} \rho C_{x} C_{y}\right)-2\left(1-\alpha_{i}\right) \gamma_{i}\left(1+\theta_{i} \rho C_{x} C_{y}\right)\right\} \\
& \operatorname{MSE}\left(\hat{\bar{Y}}_{P i}\right)=\bar{Y}^{2}\left(\alpha_{i} \lambda_{i}+\left(1-\alpha_{i}\right) \gamma_{i}-1\right)^{2}+\alpha_{i}{ }^{2} \lambda_{i}{ }^{2}\left\{\operatorname{MSE}\left(\hat{\bar{Y}}_{M R i}\right)+2 \bar{Y} B\left(\hat{\bar{Y}}_{M R i}\right)\right\} \\
& +\left(1-\alpha_{i}\right)^{2} \gamma_{i}^{2}\left\{M S E\left(\hat{\bar{Y}}_{M P i}\right)+2 \bar{Y} B\left(\hat{\bar{Y}}_{M P i}\right)\right\} \\
& -2 \bar{Y}\left\{\alpha_{i} \lambda_{i} B\left(\hat{\bar{Y}}_{M R i}\right)+\left(1-\alpha_{i}\right) \gamma_{i} B\left(\hat{\bar{Y}}_{M P i}\right)\right\}+2\left(\alpha_{i} \lambda_{i}\left(1-\alpha_{i}\right) \gamma_{i} \mathrm{~V}\left(\bar{y}_{s r s}\right)\right. \\
& \operatorname{MSE}\left(\hat{\bar{Y}}_{P i}\right)=\bar{Y}^{2}\left(P_{i}-1\right)^{2}+\alpha_{i}{ }^{2} \lambda_{i}{ }^{2}\left\{\operatorname{MSE}\left(\hat{\bar{Y}}_{M R i}\right)+2 \bar{Y} B\left(\hat{\bar{Y}}_{M R i}\right)\right\} \\
& +\left(1-\alpha_{i}\right)^{2} \gamma_{i}^{2}\left\{M S E\left(\hat{\bar{Y}}_{M P i}\right)+2 \bar{Y} B\left(\hat{\bar{Y}}_{M P i}\right)\right\} \\
& -2 \bar{Y}\left\{\alpha_{i} \lambda_{i} B\left(\hat{\bar{Y}}_{M R i}\right)+\left(1-\alpha_{i}\right) \gamma_{i} B\left(\hat{Y}_{M P i}\right)\right\}+2\left(\alpha_{i} \lambda_{i}\left(1-\alpha_{i}\right) \gamma_{i} \mathrm{~V}\left(\bar{y}_{s r s}\right)\right.
\end{aligned}
$$

We take a substitution $P_{i}=\left(\alpha_{i} \lambda_{i}+\left(1-\alpha_{i}\right) \gamma_{i}\right)$ and $Q_{i}=\left(\alpha_{i} \lambda_{i}-\left(1-\alpha_{i}\right) \gamma_{i}\right)$ then the MSE is

$$
\begin{aligned}
M S E\left(\hat{\bar{Y}}_{P i}\right)= & \bar{Y}^{2}\left(P_{i}-1\right)^{2}+\frac{\left(P_{i}+Q_{i}\right)^{2}}{4}\left\{M S E\left(\hat{\bar{Y}}_{M R i}\right)+2 \bar{Y} B\left(\hat{\bar{Y}}_{M R i}\right)\right\} \\
& +\frac{\left(P_{i}-Q_{i}\right)^{2}}{4}\left\{M S E\left(\hat{\bar{Y}}_{M P i}\right)+2 \bar{Y} B\left(\hat{\bar{Y}}_{M P i}\right)\right\} \\
& \left.-2 \bar{Y}\left\{\frac{\left(P_{i}+Q_{i}\right)}{2} B\left(\hat{\bar{Y}}_{M R i}\right)+\frac{\left(P_{i}-Q_{i}\right)}{2} B\left(\hat{\bar{Y}}_{M P i}\right)\right\}+2 \frac{\left(P_{i}^{2}-Q_{i}{ }^{2}\right)}{4} \mathrm{~V}\left(\bar{y}_{s r s}\right)\right] \\
\operatorname{MSE}\left(\hat{\bar{Y}}_{P i}\right)= & \frac{1}{4}\left[4 \bar{Y}^{2}\left(P_{i}-1\right)^{2}+\left(P_{i}+Q_{i}\right)^{2}\left\{M S E\left(\hat{\bar{Y}}_{M R i}\right)+2 \bar{Y} B\left(\hat{\bar{Y}}_{M R i}\right)\right\}\right. \\
& +\left(P_{i}-Q_{i}\right)^{2}\left\{M S E\left(\hat{\bar{Y}}_{M P i}\right)+2 \bar{Y} B\left(\hat{\bar{Y}}_{M P i}\right)\right\} \\
& \left.-4 \bar{Y}\left\{\left(P_{i}+Q_{i}\right) B\left(\hat{\bar{Y}}_{M R i}\right)+\left(P_{i}-Q_{i}\right) B\left(\hat{\bar{Y}}_{M P i}\right)\right\}+2\left(P_{i}{ }^{2}-Q_{i}{ }^{2}\right) \mathrm{V}\left(\bar{y}_{s r s}\right)\right]
\end{aligned}
$$




$$
\begin{gathered}
\text { WhereB }\left(\hat{\bar{Y}}_{M R i}\right)=\delta \bar{Y}\left[\theta_{i}{ }^{2} C_{x}{ }^{2}-\theta_{i} \rho \mathrm{C}_{\mathrm{x}} \mathrm{C}_{\mathrm{y}}\right], \mathrm{B}\left(\hat{\bar{Y}}_{M P i}\right)=\delta \bar{Y}\left[\theta_{i} \rho \mathrm{C}_{\mathrm{x}} \mathrm{C}_{\mathrm{y}}\right] \\
\operatorname{MSE}\left(\hat{\bar{Y}}_{M R i}\right)=\delta \bar{Y}^{2}\left[C_{y}{ }^{2}+\theta_{i}{ }^{2} C_{x}{ }^{2}-2 \theta_{i} \rho \mathrm{C}_{\mathrm{x}} \mathrm{C}_{\mathrm{y}}\right] \\
\operatorname{MSE}\left(\hat{\bar{Y}}_{M P i}\right)=\delta \bar{Y}^{2}\left[C_{y}{ }^{2}+\theta_{i}{ }^{2} C_{x}{ }^{2}+2 \theta_{i} \rho \mathrm{C}_{\mathrm{x}} \mathrm{C}_{\mathrm{y}}\right] \operatorname{andV}\left(\bar{y}_{s r s}\right)=\delta S_{y}{ }^{2}=\delta \bar{Y}^{2} C_{y}{ }^{2} \\
\delta=\left(\frac{1-\mathrm{f}}{\mathrm{n}}\right), \theta_{1}=\frac{C_{x} \bar{X}}{C_{x} \bar{X}+\beta_{1}}, \theta_{2}=\frac{\beta_{1} \bar{X}}{\beta_{1} \bar{X}+C_{x}} \theta_{3}=\frac{C_{x} \bar{X}}{C_{x} \bar{X}+\rho}, \theta_{4}=\frac{\rho \bar{X}}{\rho \bar{X}+C_{x}}, \theta_{5}=\frac{C_{x} \bar{X}}{C_{x} \bar{X}+\beta_{2}}, \theta_{6}=\frac{\beta_{2} \bar{X}}{\beta_{2} \bar{X}+C_{x}}
\end{gathered}
$$

where $, \mathrm{i}=1,2,3,4,5,6$

The optimal value of $\alpha_{i}$ is determined by minimize the MSE $\left(\hat{\bar{Y}}_{p i}\right)$ with respect to $\alpha_{i}$. For this differentiate MSE with respect to $\alpha_{i}$ and equate to zero.

$$
\begin{aligned}
& \text { ie, } \frac{\partial M S E}{\partial \alpha_{i}}=0 \text {, and we get the value of } \alpha_{i} \text {, as } \\
& \left.2 \bar{Y}^{2}\left(\alpha_{i} \lambda_{\mathrm{i}}+\left(1-\alpha_{i}\right) \gamma_{i}-1\right)\left(\lambda_{\mathrm{i}}-\gamma_{i}\right)+2 \alpha_{i} \lambda_{i}^{2}\left\{\operatorname{MSE}\left(\hat{\bar{Y}}_{M R i}\right)+2 \bar{Y} B\left(\hat{\bar{Y}}_{M R i}\right)\right\}\right) \\
& \left.-2\left(1-\alpha_{i}\right) \gamma_{i}^{2}\left\{M S E\left(\hat{\bar{Y}}_{M P i}\right)+2 \bar{Y} B\left(\hat{\bar{Y}}_{M P i}\right)\right\}\right)-2 \bar{Y}\left\{\lambda_{i} B\left(\hat{\bar{Y}}_{M R i}\right)+\gamma_{i} B\left(\hat{\bar{Y}}_{M P i}\right)\right\} \\
& +2\left(\lambda_{i}\left(1-2 \alpha_{i}\right) \gamma_{i} \mathrm{~V}\left(\bar{y}_{s r s}\right)=0\right. \\
& \left(\bar{Y}^{2}\left(\gamma_{i}-1\right)\left(\gamma_{i}-\lambda_{i}\right)+\gamma_{i}^{2}\left\{M S E\left(\hat{Y}_{M P i}\right)+2 \bar{Y} B\left(\hat{Y}_{M P i}\right)\right\}+\bar{Y}\left\{\lambda_{i} B\left(\hat{Y}_{M R i}\right)-\gamma_{i} B\left(\hat{Y}_{M P i}\right)\right\}\right. \\
& \alpha_{i}=\frac{\left.-\lambda_{i} \gamma_{i} \mathrm{~V}\left(\bar{y}_{S r S}\right)\right)}{\left(\bar{Y}^{2}\left(\lambda_{i}-\gamma_{i}\right)^{2}+\lambda_{i}{ }^{2}\left\{M S E\left(\hat{\bar{Y}}_{M R i}\right)+2 \bar{Y} B\left(\hat{\bar{Y}}_{M R i}\right)\right\}+\gamma_{i}{ }^{2}\left\{M S E\left(\hat{\bar{Y}}_{M P i}\right)+2 \bar{Y} B\left(\hat{\bar{Y}}_{M P i}\right)\right\}\right.} \\
& \left.-2 \lambda_{i} \gamma_{i} \mathrm{~V}\left(\bar{y}_{s r s}\right)\right)
\end{aligned}
$$

Where $\delta=\left(\frac{1-\mathrm{f}}{\mathrm{n}}\right), \mathrm{i}=1,2,3,4,5,6$ 